\title{
Activated Macrophage and Granulomatous Inflammation
}

\author{
Bareerah Zahoor, Fatima Khan, and Mehwish Azhar* \\ Department of Pharmacy, Benazir Bhutto Shaheed University, Pakistan
}

Submission: June 15, 2021; Published: October 23, 2021

*Corresponding author: Mehwish Azhar, Department of Pharmacy, Benazir Bhutto Shaheed University, Karachi, Pakistan

\section{Abstract}

The granulomatous inflammation is a characteristic type of chronic inflammation in which main cells are macrophages, multinucleated cells and epithelioid cells. The dynamic structure of granuloma helps to defend the body from microbiological threats. Granulomatous inflammation is a histologic pattern of tissue reactions which appears following cell damage. A variety of systemic diseases are occurred by this special inflammatory response. The granulomatous inflammatory response is common in many infective, allergic, autoimmune, toxic and neoplastic diseases. Mechanism that regulates granulomatous inflammation is remain not properly understood. The pulmonary system is one of the most common concerned sites to face the granulomatous inflammation. Unlike the lungs, skin can be affected by different ways including direct inoculation, endogenous origins and hematogenous spread. In this theory, we discuss about formation of granuloma, and that the granulomatous inflammation is a protective mechanism of our body but sometimes it can also cause serious and severe diseases in different body organs.

Keywords: Macrophages; Epithelioid cells; Multinucleated giant cells; Granuloma; Granulomatous inflammation

Abbreviations: GIN: Granulomatous Interstitial Nephritis; TB: Tuberculosis; NTM: Non-Tuberculous Mycobacterium

\section{Introduction}

Granulomatous inflammation is a special type of chronic inflammation. In response to different infectious agents such as bacteria or fungi etc. It can occur in these macrophages, epithelioid cells and multinucleated giant cells are also involved, which are formed from mononuclear phagocytic system. These cells are arranged in special form to form a unique structure called granuloma. In this granuloma other than these cells, some other cells like lymphocytes, plasma cells and fibroblast are also present. Eosinophils are present in the fungal and parasitic granuloma [1]. Newly arrived monocyte are initially simple cells that produce prominent nucleoli, extensive cytoplasm, free ribosomes, abundant Golgi apparatus, massive lysosomes, and eventually take on the morphology of activated macrophages.

Macrophages develop from bone marrow precursors through circulating monocytes, move through a maturation process that includes morphological and functional changes. This process continues even after macrophages reach tissues, where they are known as histiocytes [2]. Monocyte production is regulated by feedback; peripheral macrophages and lymphocytes secrete factors that have both stimulating and inhibitory effects on bone marrow stem cell proliferation. Despite the fact that exudation appears to be the primary source of macrophages in the inflammatory response, local histiocytic proliferation does occur [3].

\section{Macrophages}

Macrophages are the effector cells of innate immunity and act on the adaptive immune system in the potent effector mechanism. In bone marrow, monocytes formed from stem cells, then circulate into blood and when inflammation initiate, monocytes mature and convert into macrophages and start to perform their functions [4].

\section{Epithelioid cells}

The activated macrophages transformed into epithelioid appearance which are flattened oval shape, ovoid nuclei and membranes that attached with the adjacent cells. Epithelioid cells are the part of mononuclear phagocytic system that is found in granuloma, associated with the intense immunological activity and it has a little phagocytic activity [5]. 


\section{Granuloma}

Granuloma is a focal, compact mass of inflammatory cells in which the mononuclear cells are predominant [6]. Granuloma is normally the result of protective mechanism and formed when acute inflammatory processes can't destroy the invading agents [7]. Granuloma formations start from any inflammatory trigger/ stimulus like infectious pathogens or site of inflammation and activated as it is the part of innate immune response.

If activated macrophages are unable to remove inflammatory response, further immune response can be stimulated that are orchestrated by the dendrite cells and major histocompatibility complex II. Macrophages are increased at the site of inflammation and chronic inflammation mechanism develops. The macrophages start to form the tight aggregation around the inflammatory stimulus and become surrounded by peripheral cuff of lymphocytes. This structure or inflammatory focus is called granuloma [8].

\section{Granulomatous Inflammation}

Granulomatous inflammation appears at the cell injury. It is a histologic pattern of tissue reactions. It is caused by the different conditions like infection, drug, neoplastic, autoimmune, allergy, and toxic conditions [9]. This specialized inflammatory process is a last expedient when other immune protective mechanisms are unable to eradicate inflammatory stimulus. The mature macrophages (epithelioid cells, giant cells) aggregate around the antigen and form granuloma.

\section{Granulomas are the Site of Macrophage Activation}

Granulomatous inflammation is a form of chronic inflammation characterized by a dense array of mononuclear phagocyte system cells, primarily activated macrophages and cells derived from them [3]. The term activated macrophage denotes either a rise in the macrophage's functional activity or the appearance of a new functional activity. Newly arrived monocyte are initially simple cells that produce prominent nucleoli, extensive cytoplasm, free ribosomes, abundant Golgi apparatus, massive lysosomes, and eventually take on the morphology of activated macrophages.

Macrophages develop from bone marrow precursors through circulating monocytes, move through a maturation process that includes morphological and functional changes. This process continues even after macrophages reach tissues, where they are known as histiocytes. Monocyte production is regulated by feedback; peripheral macrophages and lymphocytes secrete factors that have both stimulating and inhibitory effects on bone marrow stem cell proliferation. Even though exudation appears to be the primary source of macrophages in the inflammatory response, local histiocytic proliferation does occur [10].

\section{Activation of Macrophages}

Activation of macrophage occurs in two steps in the case of transplantation. Firstly, macrophages become activated as a result of tissue injury caused by ischemia-reperfusion, resulting in easily graft damage. Then, in response to danger signals provided by the mismatched transplant, they become triggered. Macrophages have remarkable plasticity, allowing them to react to environmental signals and alter their phenotype quickly, with M1 macrophage on one extreme and M2 macrophages on the other [11].

Chemically activated macrophages are classified as M1, whereas alternatively activated macrophages and all the other forms are classified as M2. Alternatively activated macrophages can counteract the release of pro-inflammatory cytokines by classically activated macrophages during transplantation enabling tissue homeostasis to be restored. Later on, a subset of macrophages known as regulatory macrophages can dampen proinflammatory immune responses.

\section{Disease Caused by Granuloma}

Although granulomas are better to prevent the disease [12], but granulomatous bodies can be further divided into organ systems that are majorly affected, such as the lungs, skin, kidney, liver and lymph nodes.

\section{Effects on Different Organs}

\section{Lungs}

Infectious lung diseases are a common cause of pulmonary granulomas, so it's crucial to rule them out [13]. In pulmonary granulomas, mycobacteria and fungi are the most common species discovered. While both necrotizing and non-necrotizing granulomas can be seen in infectious lung diseases, necrotizing granulomas are commonly seen. Clinicians should be aware that, depending on the patient's tuberculosis (TB) can also manifest as non-necrotizing granulomas. Tuberculosis and non-tuberculous mycobacterial infections appear in different ways are the most common causes of necrotizing granuloma.

\section{Mycobacterium tuberculosis}

Mycobacterium tuberculosis is an acid-fast bacillus. Inhalation of this bacterium causes a histologic granulomatous response in the lungs, which is mediated by alike cytokine pathways. The chronic granulomatous response controls inflammation by activating histiocytes and forming a rim of histiocytes and peripheral lymphocytes.

\section{Non-Tuberculous Mycobacterium}

NTM are mycobacterial organisms that are not part of the mycobacterium tuberculosis complex. Previously, it was believed that NTM infection in the lungs was linked to immunodeficiency or 
preexisting lung disease, such as chronic obstructive pulmonary disease or cystic fibrosis. However, it is known that NTM infection of the lungs can occur in immunocompetent patients who do not have any prior lung disease.

\section{Skin}

Granulomatous skin disorder caused by pathogens and these pathogens may identify by used of special tissue stains [14]. Epithelioid granulomas are made up of epithelioid histiocytic or macrophages, some of which combine to form massive multinucleated giant cells, lymphocytes, and sometimes plasma cells with or without necrosis. Mycobacterium leprae causes leprosy, a chronic infectious granulomatous disease that is common in many parts of the world. Leprosy may affect the skin and peripheral nerves, with macular, nodular, infiltrative, or diffuse lesions being the most common clinical manifestations. It has different histopathological appearances depending on the host's immunity [15].

\section{Kidney}

Renal failure or renal disorder is may also cause by granulomatous interstitial nephritis (GIN) [16], a rare condition. Sarcoidosis, granulomatous with polyangiitis, and infectious agents are the most common causes (particularly renal tuberculosis). Noncaseating granulomatous inflammation, which is made up of a central follicle of macrophages, epithelioid cells, and multinucleated giant cells, is thought to be the pathology that contributes to AKI from GIN in sarcoidosis.

\section{Liver}

In the liver, due to the activation of the immune system by a variety of agents, granulomas, which are focal accumulations of macrophages, are typically located in the liver [17]. Symptoms are different which Depend on whether the underlying cause is a systematic disorder or a primary hepatic granulomatous reaction. Granulomas can form or collect in the liver near portal tracts and are caused to increase in alkaline phosphatase. As seen in sarcoidosis, cells inside the granuloma can secrete a variety of proteins and enzymes such as lysozyme, collagenase, and angiotensin-converting enzyme. Necrosis in different types may also reveal information about the cause of granulomas. These results include 'caseating' necrosis, as in pulmonary tuberculosis, and 'non caseating' necrosis, as in sarcoid granulomas.

\section{Discussion}

Granulomatous inflammation is a histologic way of tissue reaction that happens after some cell injury. Granulomatous inflammation is cause by many conditions that may include infection, autoimmune, allergic, toxins, drug, and some other neoplastic conditions. The tissue reaction swatch reduces some of the pathologic and clinical diagnosis and clinical deception. Most of the reaction ways are necrotizing granulomas, non necrotizing granulomas, suppurative granulomas, diffuse granulomatous inflammation, and foreign body large cell reaction.

Prototypical examples of necrotizing granulomas are observed with mycobacterial infections and non-necrotizing granulomas with sarcoidosis. The pulmonary system is one of the most commonly affected way to face granulomatous inflammation. Infectious causes of granuloma are most common. Unlike the lung, skin can be affected by many ways, which may include direct inoculation, endogenous sources, and hematogenous spread. This involvement may interact a variety of infectious agents, which can present as necrotizing or non-necrotizing granulomatous inflammation.

Non-infectious etiologies need a clinicopathologic summarization to reduce the scope of the pathogenesis which include foreign body reaction, autoimmune, neoplastic, and drug related etiologies. Granulomatous inflammation of the kidney, often referred to as granulomatous interstitial nephritis (GIN) is unlike organ systems such as the skin or lungs. The differential diagnosis of GIN is more frequently due to drugs and sarcoidosis as compared to infections (fungal and mycobacterial).

\section{Conclusion}

Granulomatous inflammation is a form of chronic inflammation characterized by a dense array of mononuclear phagocyte system cells, primarily activated macrophages and cells are derived from them. The term activated macrophage denotes either a rise in the macrophage's functional activity or the appearance of a new functional activity. The aim of this granuloma is to isolate the antigen from the body and facilitate its eradication to protect the body from any type of infection or injury or disease. Granulomas are structured aggregates of macrophages and other immune cells, often with distinct morphological changes. The granulomatous response is a stepwise process marked by a series of macrophage activations and transformations, which recruit additional cells and cause structural changes. Granulomas evolved as defensive responses to kill or sequester particles, but they are often pathological when foreign bodies, infections, or inflammatory diseases are present.

\section{References}

1. GT Williams, WJ Williams (1983) Granulomatous inflammation--a review. J Clin Pathol 36(7): 723-33.

2. Julien V, Alexis D, Antoine D, Nelly B, Pierre C, et al. (2017) A Role for CD154, the CD40 Ligand, in Granulomatous Inflammation. Mediators of Inflammation P: 14.

3. T de Brito, MF Franco (1994) Granulomatous inflammation. Rev Inst With trop S Paulo 36 (2): 185-192.

4. AA Alhassani, MS Al-Zahrani, KH Zawawi (2020) Granulomatous diseases: Oral manifestations and recommendations. Saudi Dent J 32(5): 219-223.

5. JL Turk, RB Narayanan (1982) The Origin, Morphology, and Function of Epithelioid Cells. Immunobiology 161(3-4): 274-282. 
6. BN Kumarguru, M Natarajan, Dayananda SB, AR Raghupathi (2015) Giant Cell Lesions of Lungs: A Histopathological and Morphometric Study of Seven Autopsy Cases. J Clin Diagn Res 9(11): EC12-6.

7. A Zumla, DG James (1996) Granulomatous Infections: Etiology and Classification. Clinical Infectious Diseases 23(1): 146-158.

8. Olivia W, Saira F (2021) Granuloma. StatPearls (Internet).

9. KK Shah, BS Pritt, MP Alexander (2017) Histopathologic review of granulomatous inflammation. J Clin Tuberc Other Mycobact Dis 7: $1-12$.

10. Helen JP, Andrew MS (2013) The role of the innate immune system in granulomatous disorders. Front Immunol 4:120.

11. https://www.sciencedirect.com/topics/medicine-and-dentistry/ macrophage-activation

12. AJ Pagan, L Ramakrishnan (2018) The Formation and Function of Granulomas. Annual Review of Immunology 36: 639-665.
13. Shinichiro O, Josune G, U Costabel, Francesco B (2017) Differential diagnosis of granulomatous lung disease: clues and pitfalls. European Respiratory Review 26(145): 170012.

14. Christina M, Michael T, (2016) Histologic features of granulomatous skin diseases. JDDG 14(4): 378-387.

15. Rajeshwari K, Nandkumar D, KG Nagappa, C Rokade (2016) Infectious Granulomatous Dermatitis at a Tertiary Care Centre in North Maharashtra: A Histopathological Study. J Clin Diagn Res 10(11): EC13EC16.

16. S Sharmeen, E Kalkan, Chunhui Y, Steven DS (2016) Granulomatous Interstitial Nephritis Presenting as Hypercalcemia and Nephrolithiasis. Case Rep Nephrol 2016: 4186086.

17. M Coash, F Forouhar, CH Wu, GY Wu (2012) Granulomatous liver diseases: A review. Journal of the Formosan Medical Association 111(1): 3-13.

Your next submission with Juniper Publishers will reach you the below assets

- Quality Editorial service

- Swift Peer Review

- Reprints availability

- E-prints Service

- Manuscript Podcast for convenient understanding

- Global attainment for your research

- Manuscript accessibility in different formats (Pdf, E-pub, Full Text, Audio)

- Unceasing customer service

Track the below URL for one-step submission https://juniperpublishers.com/online-submission.php 